\title{
A comparative study of the susceptibility of cut and uncut enamel to erosive demineralization
}

\author{
Wa Than LIN¹, Yuichi KITASAKO ${ }^{1,2}$, Syozi NAKASHIMA ${ }^{1}$ and Junji TAGAMI ${ }^{1}$ \\ ${ }^{1}$ Cariology and Operative Dentistry, Department of Restorative Sciences, Graduate School of Medical and Dental Sciences, Tokyo Medical and \\ Dental University, 1-5-45 Yushima, Bunkyo-ku, Tokyo 113-8549, Japan \\ ${ }^{2}$ Dental clinic, Ministry of Foreign Affairs of Japan, 2-2-1 Kasumigaseki, Chiyoda-ku, Tokyo 100-8919, Japan \\ Corresponding author, Yuichi KITASAKO; E-mail: kitasako.ope@tmd.ac.jp
}

\begin{abstract}
This study aimed to evaluate the susceptibility of cut and uncut enamel surfaces to an erosive challenge and to examine the resultant characteristics/morphological changes. Ten extracted human incisors were used for preparation of enamel samples, and samples were immersed in citric acid. After 3 (total $3 \mathrm{~min}$ ) and 6 cycles (total $6 \mathrm{~min}$ ) of erosive challenges, surface loss (SL) and morphological changes were measured using scanning microscopy and FIB-TEM. Ca release (CA) and surface hardness (SH) were measured using a calcium-sensitive electrode and hardness tester respectively. Mean values of all measurements were statistically analyzed by using a $t$-test. Uncut enamel samples had significantly lower SL and greater SH than cut enamel $(p<0.01)$. Cut enamel samples after 3 cycles showed higher CA compared with those from uncut enamel samples $(p<0.05)$. Cut enamel was shown to be more susceptible to acidic dissolution and deeper acid penetration than uncut enamel after erosive demineralization.
\end{abstract}

Keywords: Enamel, Erosion, Surface hardness, Surface loss, FIB-TEM

\section{INTRODUCTION}

Dental erosion is defined as the pathologic, chronic, localized irreversible loss of dental hard tissue caused by the exposure of tooth surfaces to extrinsic or intrinsic acids that does not involve bacteria ${ }^{1)}$. Dental erosion has become a significant problem due to the increased consumption of soft drinks worldwide with changing life style and easy availability of erosive drinks and foods ${ }^{2,3}$. Various acidic soft drinks, juices and fruits can cause a decrease in the surface hardness of tooth enamel ${ }^{4)}$. Also the prevalence of dental erosion has increased in recent years, especially in the young age group ${ }^{2,5)}$.

When the enamel surface is exposed to an acidic solution, hydroxyapatite is dissolved with a subsequent loss of calcium and phosphate from apatite crystals ${ }^{6}$. Continuous acid attack will lead to an increased loss of minerals and subsequent softening of enamel surface. The mineral content in enamel can differ depending on the tooth anatomy and age ${ }^{7}$. The fluoride, calcium and phosphorus contents and enamel density tend to decrease from the surface to the inner region ${ }^{7-9)}$, while the carbonate and magnesium concentrations increase towards the dentin-enamel junction $(\mathrm{DEJ})^{10}$. These differences in mineral gradient can be used to describe about how mineral dissolution from the surface to DEJ can vary ${ }^{11)}$. These variations of mineral in enamel should also be considered for the use of enamel laboratory experiments ${ }^{12)}$.

Many laboratory studies on dental erosion have focused on the chemical aspects of the erosive potential of acidic foods and beverages. In those erosion related studies, polished enamel samples were used to reduce the possible influence of superficial variations such as high fluoride content that may influence remineralization and demineralization cycles, and different ion concentrations ${ }^{13,14)}$. Carvalho and Lussi published results on the susceptibility of dental enamel to erosive dissolution in relation to different types and surfaces of teeth, as well as the enamel depth by using polished enamel samples. The tested enamel had different susceptibilities to erosion in relation to different depths from the surface ${ }^{12)}$. Ganss et al. also performed a study on the susceptibility of enamel, coronal and root dentin to acidic dissolution that included polished and natural enamel samples. In the enamel samples, they showed different surfaces had different susceptibility to an erosive solution ${ }^{15)}$. However, there is less information on the erosive susceptibility of the superficial layer that is more resistant to acid erosion than subsurface enamel ${ }^{15,16)}$.

There is limited information on the understanding of susceptibility of natural enamel surfaces to erosive challenges in intra oral conditions using various qualitative assessments. To understand the progression of erosion under intraoral conditions, the aim of the present study was to evaluate the susceptibility of cut and uncut enamel surfaces to an erosive challenge and to examine the resultant characteristics/morphological changes. The null hypothesis was that there were no differences in the susceptibilities of laboratory dissolution (erosive challenge) between cut and uncut enamel surfaces.

\section{MATERIALS AND METHODS}

\section{Enamel sample preparation}

Ten freshly extracted, non-damaged human incisors (age 43-75 years) were used for preparation of enamel samples. The teeth were cleaned to remove any residual 
tissues and the root was separated from the crown. The labial surface of the crown was divided into two enamel samples by sectioning with a slow speed diamond saw (Isomet, Buehler, IL, USA) under constant water cooling. One sample was used as the 'uncut' group (natural enamel, $n=10$ ) and the other was ground to obtain a polished surface for the cut group (polished enamel, $n=10$ ). To obtain flat smooth polished surfaces, the specimens were ground with 800-, 1200-, 2000-grit silicon carbide papers and finished with diamond pastes down to $1 \mu \mathrm{m}$ using an automatic lapping machine (ML160A, Maruto, Tokyo, Japan) under running water. To re-trace areas precisely, two lines to define the erosive area were made by using a blade on each specimen. All specimens were covered with an adhesive tape leaving an approximately $3 \times 3 \mathrm{~mm}$ window.

\section{Erosive challenge}

All enamel samples were cleaned ultrasonically (Delta ${ }^{\circledR}$ Mini super ultrasonic cleaner, Kenis, Japan) in deionized water for $30 \mathrm{~s}$ before each surface profile measurement. Surface tracing prior to acid challenge was done for erosive surface loss measurement. Each of the samples was subjected to an acid challenge in $6 \mathrm{~mL}$ of $1 \%$ Citric acid solution ( $\mathrm{pH}$ 3.6) at room temperature for $60 \mathrm{~s}$. The sample was then rinsed with deionized water for $60 \mathrm{~s}$ and dried with air for $5 \mathrm{~s}$. The erosive challenge and rinsing occurred three times and was regarded as 1 erosive challenge cycle. After 3 cycles of erosive challenge, $3 \mathrm{~mL}$ of test (erosion) solution was retained for measurement of calcium release. The next 3 cycles were completed with the remaining $3 \mathrm{~mL}$ of test solution. After 3 (total $3 \mathrm{~min}$ ) and 6 cycles (total $6 \mathrm{~min}$ ) of erosive challenges, surface loss (SL) and surface characteristics/ morphological changes of non-eroded and eroded parts of each sample were measured using Focus Variation 3D scanning Microscopy (FVM) and FIB-TEM. Ca release $(\mathrm{CA})$ and Surface hardness $(\mathrm{SH})$ were measured by using a calcium-sensitive electrode and Cariotester respectively.

\section{Surface loss measurement}

Prior to measurement, the tape was removed from each end of the sample using forceps. The FVM (Infinite Focus G5 Microscope, Alicona Imaging, Grambach, Austria) was used to measure surface loss, surface characteristics/morphological changes and to capture 3D images of the eroded enamel surfaces. The measurement was performed using a 50× objective at a vertical resolution of $50 \mathrm{~nm}$ and lateral resolution of 2 $\mu \mathrm{m}$. To measure erosive surface loss, the surface profile of the selected area on the enamel surface was captured before and after surface treatment at the same position. The two 3D-data sets were then aligned to each other to evaluate the differences. Profile tracing was done approximately in the middle of defined area to avoid surface irregularities. The vertical distance between the highest and lowest point within the defined erosive area of each specimen was calculated. 3D images were also captured to obtain visual surface changes.

\section{Calcium released measurement}

The amount of calcium released after erosive challenge was measured using a combination type calciumsensitive electrode (6583; HORIBA, Kyoto, Japan). The electrode was connected to an ion meter (F-53; HORIBA). The Ca electrode was calibrated using standard solutions (1.5, 3.0, 6.0, 10, 30 and $50 \mathrm{ppm})$ that were prepared using $\mathrm{CaCl}_{2}$. An excellent linear equation between the $\log (\mathrm{Ca})$ concentration and corresponding $m V$ readings was obtained with $R^{2}$ being 1.00. Actual $m V$ values were observed in this concentration range. Before measurement, the test solutions from samples and standard solutions were adjusted to $\mathrm{pH} 5$ with $\mathrm{KOH}$. The released Ca amount $\left(\mu \mathrm{g} / \mathrm{mm}^{2}\right)$ was then calculated from the ppm concentration, volume of the sample solution and the erosive surface areas of all samples.

\section{Surface hardness measurement}

Cariotester (SUK-971, SaneiME, Yokohama, Japan) Fig. 1(a) was used to measure the surface hardness in order to compare the hardness of non-eroded and eroded enamel surfaces on both the cut and uncut specimens. A handpiece fitted with a cone-shaped indenter that was covered with a white colored paint at the tip was applied perpendicularly to the test surface manually using a 150 gram force load. The distance from the indenter tip to the line where the paint had disappeared (CT depth) was measured via a microscope on a computer screen Fig. 2(b). CT depth value was then converted to KHN with the programmed software. The mean hardness value was derived from five indentations done randomly across each test surface.

\section{Focused ion beam (FIB)-TEM}

Focused ion beam is a technique for site-specific analysis of materials, it operates the same way as SEM but the difference is using ions instead of electrons. The FIB system consisted of a vacuum chamber, liquid metal ion source, ion collimation, sample stage, detectors, probes, gas delivery system and computer. A liquid Gallium (Ga) metal ion source was connected to a tungsten needle. When the needle tip was connected to a high electric current, metal ions were released from the tip. The liquid Ga continuously flowed to the tip resulting in a constant ion current for the milling process. Before milling, the target area was covered with a tungsten as protective layer. Prism-shaped microsamples were ablated from the specimen. After milling was completed, the extracted micro-sample was fixed on a compatible FIB/TEM stage and ultra-thinned into $100 \mathrm{~nm}$. The specimen preparations were done by using FIB system (FB2200, Hitachi, Tokyo, Japan) and the test section was examined with TEM (HD-2700, Hitachi) for morphological analysis.

\section{Statistical analysis}

Mean values of comparison between cut and uncut samples after 3 and 6 cycles of erosive challenge in all measurements was statistically analyzed by using a $t$-test and each group was analyzed by paired $t$-tests. 

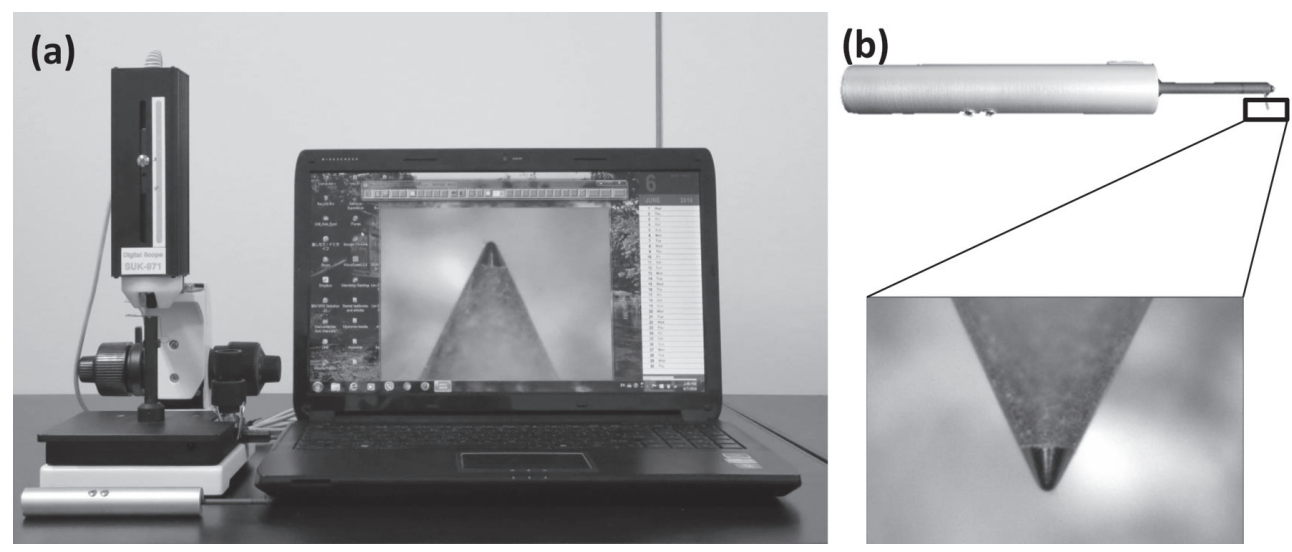

Fig. 1 (a) Overview of the Cariotester system including the digital microscope connected to a laptop computer and handpiece, (b) the distance from the indenter tip to the paint line (CT depth) after measurement gives the depth penetration measurement.

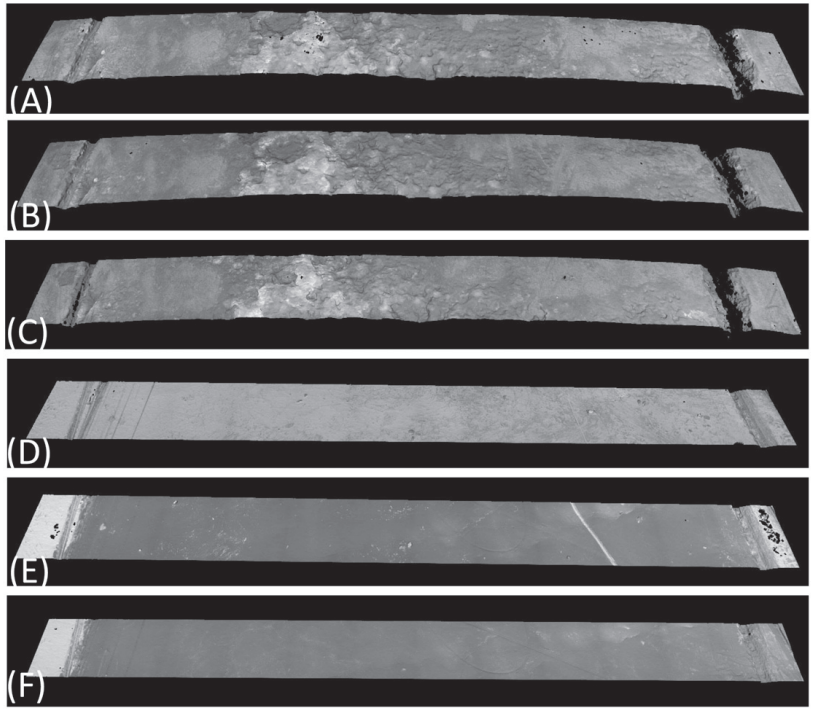

Fig. 2 FVM 3D images (50×) of human uncut enamel surface before erosion (A), after 3 erosion cycles (B) and 6 erosion cycles (C), human cut enamel surface before erosion (D), after 3 erosion cycles (E) and 6 erosion cycles (F).

The data were normally distributed (KolmogorovSmirnoff test). The level of significance was set at 0.05 alpha level. All analyses were performed by using SPSS 22 statistical software (IBM, Chicago, IL, USA).

\section{RESULTS}

Surface loss after erosive demineralization (Table 1) Uncut enamel samples were less susceptible to acidic dissolution than cut enamel samples and had lower surface loss values. There were significant differences on mean surface loss for 3 and 6 cycles between cut and uncut enamel samples $(p<0.01)$. When comparing between different cycles within the same group, significant differences were also found in both groups $(p<0.001)$.

\section{Calcium analysis (Table 1)}

Cut enamel samples after 3 cycles showed significantly higher Ca release compared with those from uncut enamel samples $(p<0.05)$. While after 6 cycles, there was no statistical difference between cut and uncut enamel $(p>0.05)$. Within both cut and uncut groups, there were no significant differences between 3 and 6 cycles $(p>0.05)$.

\section{Surface hardness (Table 1)}

Before erosive challenges and after the 6 cycles of erosion, mean surface hardness of uncut enamel was significantly greater than cut enamel $(p<0.001)$. When the results were compared between different acidic challenges within the same group, significant differences were also found in both cut and uncut groups $(p<0.001)$.

Surface characteristics/morphological changes (Figs. 1 and 2)

The 3D images of cut and uncut enamel samples before, after the 3 and 6 cycle erosive challenge are shown in Fig. 2. In the cut enamel samples, after the 3 cycle erosive challenge morphological changes such as a dark colored area were observed and were more dramatically changed after 6 cycles. However, in the uncut enamel samples, there was no clear morphological change after 3 and 6 cycles compared with the non-eroded samples.

FIB-TEM images of cut and uncut enamel surfaces with high magnification (105,000×) before erosive challenge and after the 6 cycle erosive challenge are shown in Fig. 3. In the non-eroded area, enamel rods which are parallel to the surface could be seen in both cut and uncut enamel samples. A superficial layer can be observed on the surface of the non-eroded uncut 
Table 1 Surface loss, calcium release and surface hardness of cut and uncut enamel

\begin{tabular}{|c|c|c|c|c|c|c|}
\hline & \multicolumn{2}{|c|}{ Surface loss (nm) } & \multicolumn{2}{|c|}{ Calcium released $\left(\mu \mathrm{g} / \mathrm{mm}^{2}\right)$} & \multicolumn{2}{|c|}{ Surface hardness (KHN) } \\
\hline & 3 cycles & 6 cycles & 3 cycles & 6 cycles & No-erosion & 6 cycles \\
\hline Cut & $574.9^{\mathrm{a}, \mathrm{A}}( \pm 106.5)$ & $853.6^{\mathrm{c}, \mathrm{B}}( \pm 118.4)$ & $2.7^{\mathrm{a}, \mathrm{A}}( \pm 1.2)$ & $1.9^{\mathrm{c}, \mathrm{A}}( \pm 0.7)$ & $269.6^{\mathrm{a}, \mathrm{A}}( \pm 4.7)$ & $207.1^{\mathrm{c}, \mathrm{B}}( \pm 6.4)$ \\
\hline Uncut & $431.4^{\mathrm{b}, \mathrm{C}}( \pm 96.3)$ & $660.7^{\mathrm{d}, \mathrm{D}}( \pm 124.7)$ & $1.7^{\mathrm{b}, \mathrm{B}}( \pm 0.7)$ & $1.3^{\mathrm{c}, \mathrm{B}}( \pm 0.5)$ & $313.6^{\mathrm{b}, \mathrm{C}}( \pm 6.2)$ & $263.6^{\mathrm{d}, \mathrm{D}}( \pm 5.7)$ \\
\hline
\end{tabular}

Data are presented as mean \pm SD.

Within each measurement, in each row, values with different upper case letter are statistically different $(p<0.05)$; in each column, values with different lower case letter are significantly different $(p<0.05)$.

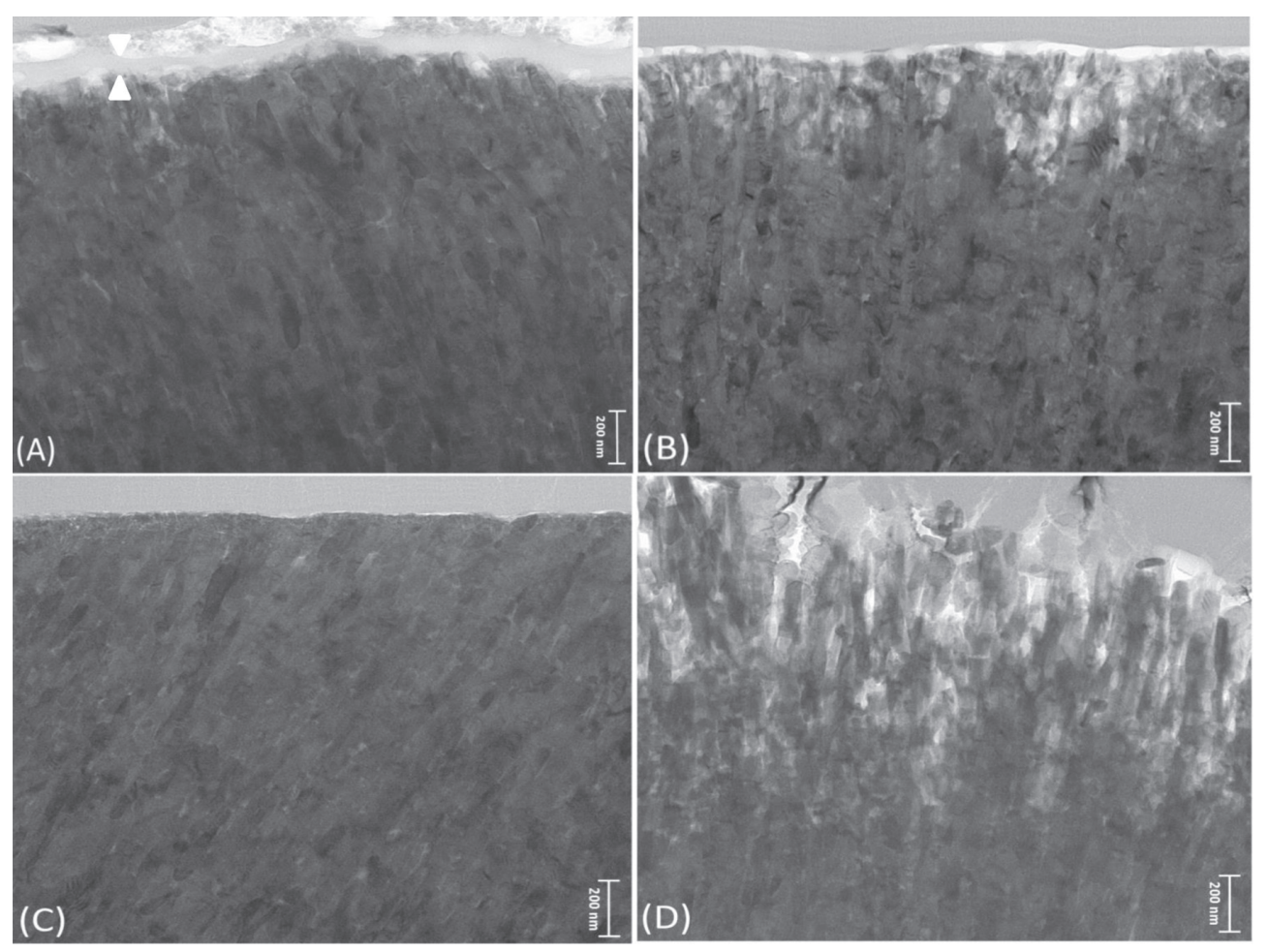

Fig. 3 FIB-TEM images of human enamel with 105,000 magnification in the vertical direction. Uncut enamel surface from non-erosion area (The structure between the arrows represents the superficial layer) (A), after 6 cycles of acidic erosion region (B), cut enamel surface from non-erosion region (C) and after 6 cycles of acidic erosion region (D).

enamel samples. After 6 cycles of erosion, the superficial layer became thinner in the uncut enamel sample. Acid penetration within the interrod spaces can be seen but the penetration pattern was irregular. Some regions showed deep penetration (500 nm) but not as deep as those (more than $1 \mu \mathrm{m}$ ) after 6 erosive cycles on the cut enamel. Enamel rods are perpendicular to the surface and the enamel crystals dissolved from the rods in some areas of the eroded cut enamel.

\section{DISCUSSION}

Human molar and premolar teeth are the most common teeth used in erosion studies whereas using incisors as the substrate seems rare ${ }^{12,15,17)}$. Upper incisors have a high incidence and prevalence to develop erosive tooth loss ${ }^{18)}$. Based on epidemiological studies, dental erosion is commonly observed on the labial surface of incisor teeth and severe tooth substance loss can be observed $^{19,20)}$. Based on this evidence, labial surfaces of human central incisors were used in this study to analyze the effects of an erosive challenge. Citric acid is a common constituent in many beverages and has a high potential to cause dental erosion because of its chelating action that attracts minerals such as calcium ions $^{4,6)}$. Different acidic beverages have different erosive effects $^{4)}$ and some contain minerals ${ }^{4,21)}$ that may interfere with chemical analysis for assessing dental erosion. To 
avoid these possible confounding problems in an erosive challenge, citric acid was used in this study.

Profilometry is used as a high precision method to assess surface loss in erosion research to enable measurement of the extent of erosion ${ }^{14)}$. FVM 3D microscopy is a powerful non-contact optical instrument that can be used to evaluate surface morphological changes without damaging the surface of samples ${ }^{17,22)}$. Recent laboratory studies suggest that there is a strong correlation between contact stylus surface profilometery (SSP) and FVM for surface roughness measurement ${ }^{22,23)}$. FVM could obtain 3D images and surface loss in the same test areas over different exposure times due to its non-damaging measurement technique.

It was shown that uncut enamel had a significantly lower surface loss and less acid dissolution than cut enamel in this study. This confirms laboratory study findings that 'natural' enamel surfaces have significantly lower erosive loss compared with polished enamel samples ${ }^{15)}$. Meurman and Frank performed a scanning electron microscope study using 'natural' human enamel, natural and polished bovine enamel samples immersed in acidic drinks. They concluded that structural factors of superficial enamel were important for progression of erosion ${ }^{16)}$. The superficial surface and presence of fluorapatite crystals which have a lower solubility than hydroxyapatite crystals of enamel were removed to some extent in the cut enamel ${ }^{6,10)}$. The rate of solubility and dissolution in enamel was also increased from the surface to the DEJ because of its mineral gradient ${ }^{11)}$. The calcium and phosphate ratio was increased at the enamel surface compared with the subsurface ${ }^{8,24)}$. The increased dissolution rate in cut enamel is likely to be due to the lack of a more highly mineralized layer and lesser amount of fluorapatite. In this study, 336 \pm 53.06 $\mu \mathrm{m}$ of superficial enamel was removed in the cut group. In most erosion studies, a larger amount of enamel was removed to make polished samples, so that the importance of uncut enamel has been disregarded.

For the accurate laboratory measurement of hardness assessment, a polished (cut) surface is essential $^{25)}$. Recently, a newly developed hardness system to measure Knoop hardness has been introduced with the advantage that it is simple, quick and can be used to measure an unpolished surface ${ }^{26}$. In this study, this device was used to measure KHN of non-eroded and eroded surfaces of cut and uncut enamel samples. For both the before and after acid erosion conditions, the uncut enamel showed significantly greater surface hardness values than the cut enamel. To our knowledge, this is the first laboratory report on the superficial enamel hardness of uncut human enamel samples. Increased surface hardness values in the uncut samples could be due to the increased mineral content, and density caused by post eruptive maturation in the enamel surface compared with the subsurface enamel ${ }^{24,27-29)}$. These hardness results suggested the importance of the superficial enamel layer of natural (uncut) enamel and is an important layer to resist acidic challenge, either that from erosive changes or caries. From the results of the high resolution FIB-TEM study, an approximately $100 \mathrm{~nm}$ thickness of the superficial layer of uncut and non-eroded enamel was observed. This superficial layer may have a mineralized layer superimposed due to repeated deposition and reposition process of minerals. However, the clinical formation of this layer was not clear. Further laboratory and in situ/in vivo studies are needed to understand the importance of the properties of the superficial layer.

Chemical analysis is a quantitative method for erosion assessment with the advantage of detecting small quantities of mineral dissolution from the eroded enamel ${ }^{14)}$. Calcium analysis is a reliable and sensitive method to assess erosion ${ }^{12,30)}$. Calcium released from cut enamel was significantly higher than uncut enamel after 3 acid challenge cycles. The solubility and dissolution of enamel mineral increased in relation to depth towards the $\mathrm{DEJ}^{11)}$. The increased calcium released from cut enamel compared with uncut enamel was similar to other laboratory studies ${ }^{11,31)}$. Polished enamel showed greater dissolution than the unpolished surface and the aprismatic enamel in natural samples showed irregular dissolution $^{16)}$. From the results of the TEM images of the eroded cut enamel, the acid could penetrate more than $1 \mu \mathrm{m}$ into the interrod spaces while in those of the uncut enamel showed irregular penetration with the greatest depth of $500 \mathrm{~nm}$. These results support the greater dissolution observed in cut enamel samples. The presence of the superficial enamel layer was able to prevent acid penetration into the subsurface region and reduce dissolution in the uncut enamel. Further study is needed to evaluate the relationship between the superficial enamel structure and its chemical composition.

However, these findings should be considered in the context of several limitations. Mineral loss from the tooth surface including calcium release was found to increase in association with increased acid challenge time $^{12,13,30,32)}$. However, Ca decreased after 6 cycles in both groups. That could be due to the calculation technique or $\mathrm{Ca}$ complexing with other ions in the solution. Using citric acid can also have limitations because of the formation of Ca complexes that impaired correct calcium release measurements from the tooth ${ }^{14)}$. Due to this limitation, it is difficult to compare the amount of Ca release between 3 and 6 cycle challenges. Lack of a detailed history of the incisors that were used in the study may also be a limitation in this research. Superficial tooth loss from previous dental treatment, root exposure due to periodontal disease, the progression of physiological and pathological tooth wear has a reduced relevance clinically ${ }^{15}$. The null hypothesis was rejected as most values were significantly different in our study between cut and uncut enamel samples.

\section{CONCLUSION}

From the results of this laboratory study, the cut enamel was shown to be more susceptible to acidic dissolution than uncut enamel which showed greater 
resistance to erosive challenges. Cut enamel shows deeper acid penetration than uncut enamel after erosion demineralization.

\section{ACKNOWLEDGMENTS}

This research was supported, in part, by a Grant-in-Aid for Scientific Research (No. 16K11541) from the Japan Society for the Promotion of Science (JSPS). The authors would like to thank to Prof. Michael BURROW, Dr. Akihiko SHIMIZU and Dr. Masaomi IKEDA for their helpful support to this study.

\section{REFERENCES}

1) ten Cate JM, Imfeld T. Dental erosion, summary. Eur J Oral Sci 1996; 104: 241-244.

2) Lussi A, Carvalho TS. Erosive tooth wear - a multifactorial condition of growing concern and increasing knowledge. Monogr Oral Sci 2014; 25: 1-15.

3) Gambon DL, Brand HS, Veerman ECI. Dental erosion in the 21st century: what is happening to nutritional habits and lifestyle in our society? Br Dent J 2012; 213: 55-57.

4) Lussi A, Megert B, Shellis RP, Wang X. Analysis of the erosive effect of different dietary substances and medications. Br J Nutr 2012; 107: 252-262.

5) Jaeggi T, Lussi A. Prevalence, incidence and distribution of erosion. Monogr Oral Sci 2014; 25: 55-73.

6) Shellis RP, Featherstone JD, Lussi A. Understanding the chemistry of dental erosion. Monogr Oral Sci 2014; 25: 163179.

7) Weatherell JA, Robinson C, Hallsworth AS. Variations in the chemical composition of human enamel. J Dent Res 1974; 53: 180-192.

8) Robinson C, Weatherell JA, Hallsworth AS. Variation in composition of dental enamel within thin ground tooth sections. Caries Res 1971; 5: 44-57.

9) Nakagaki H, Koyama Y, Sakakibara Y, Weatherell JA, Robinson C. Distribution of Fluoride across human dental enamel, dentine and cementum. Arch Oral Biol 1987; 32: 651654

10) Weatherell JA, Robinson C, Hiller CR. Distribution of carbonate in thin sections of dental enamel. Caries Res 1968; $2 ; 1-9$.

11) Thenus HM, Driessens FC, van Dijk JW, Groeneveld A. Experimental evidence for a gradient in the solubility and in the rate of dissolution of human enamel. Caries Res 1986; 20: 24-31.

12) Carvalho TS, Lussi A. Susceptibility of enamel to initial erosion in relation to tooth type, tooth surface and enamel depth. Caries Res 2015; 49: 109-115.

13) Tucker K, Adams M, Shaw L, Smith AJ. Human enamel as a substrate for in vitro acid dissolution studies: Influence of tooth surface and morphology. Caries Res 1998; 32: 135-140.

14) Attin T, Wegehaupt FJ. Methods for assessment of dental erosion. Monogr Oral Sci 2014; 25: 123-142.

15) Ganss C, Klimek J, Schwarz N. A comparative profilometric in vitro study of the susceptibility of polished and natural human enamel and dentine surfaces to erosive demineralization. Arch Oral Biol 2000; 45: 897-902.

16) Meurman JH, Frank RM. Progression and surface ultrastructure of in vitro caused erosive lesions in human and bovine enamel. Caries Res 1991; 25: 81-87.

17) Ren YF, Zhao Q, Malmstrom H, Barnes V, Xu T. Assessing fluoride treatment and resistance of dental enamel to softdrink erosion in vitro: applications of focus variation 3D scanning microscopy and stylus profilometry. J Dent 2009; 37: 167-176.

18) El Aidi H, Bronkhorst EM, Huysmans MC, Truin GJ. Factors associated with the incidence of erosive wear in upper incisors and lower first molars: A multifactorial approach. J Dent 2011; 39: 558-563.

19) Kitasako Y, Sasaki Y, Takagaki T, Sadr A, Tagami J. Agespecific prevalence of erosive tooth wear by acidic diet and gastroesophageal reflux in Japan. J Dent 2015; 43: 418-423.

20) Kitasako Y, Sasaki Y, Takagaki T, Sadr A, Tagami J. Tooth wear affected by dental erosion among different tooth types and surfaces in wide-age ranged Japanese adults. Oral Health Prev Dent 2016 (in press).

21) Hara AT, Zero DT. Analysis of the erosive potential of calciumcontaining acidic beverages. Eur J Oral Sci 2008; 116: 60-65.

22) Fujii M, Kitasako Y, Sadr A, Tagami J, Roughness and $\mathrm{pH}$ changes of enamel surface induced by soft drinks in vitro-applications of stylus profilometry, focus variation 3D scanning microscopy and micro $\mathrm{pH}$ sensor. Dent Mater J 2011; 30: 404-410.

23) Mita H, Kitasako $Y$, Takagaki $T$, Sadr A, Tagami J. Development and evaluation of a low-erosive apple juice drink with Phosphoryl-Oligosaccharides of Calcium. Dent Mater J 2013; 32: 212-218.

24) He B, Huang S, Zhang C, Jing J, Hao Y, Xiao L, Zhou X. Mineral densities and elemental content in different layers of healthy human enamel with varying teeth age. Arch Oral Biol 2011; 56: 997-1004.

25) Schlueter N, Hara A, Shellis RP, Ganss C. Methods for the measurement and characterization of erosion in enamel and dentine. Caries Res 2011; 45: 13-23.

26) Shimizu A, Nakashima S, Nikaido T, Sugawara T, Yamamoto T, Momoi Y. Newly developed hardness testing system, "Cariotester": measurement principles and development of a program for measuring Knoop hardness of carious dentin. Dent Mater J 2013; 32: 643-647.

27) Wilson PR, Beynon AD. Mineralization differences between human deciduous and permanent enamel measured by quantitative microradiography. Arch Oral Biol 1989; 34: 8588.

28) Kodaka T, Debari K, Yamada M, Kuroiwa M. Correlation between microhardness and mineral content in sound human enamel. Caries Res 1992; 26: 139-141.

29) Brudevold F, Aasenden R, Bakhos Y. A preliminary study of posteruptive maturation of teeth in situ. Caries Res 1982; 16: 243-248.

30) Rakhmatullina E, Bossen A, Höschele C, Wang X, Beyeler B, Meier C, Lussi A. Application of the specular and diffuse reflection analysis for in vitro diagnostics of dental erosion: correlation with enamel softening, roughness, and calcium release. J Biomed Opt 2011; 16: 107002.

31) Sullivan HR. The solubility of enamel surfaces. J Dent Res 1954; 33: 504-510.

32) Amaechi BT, Hicham SM, Edgar WM. Factors influencing the development of dental erosion in vitro: enamel type, temperature and exposure time. J Oral Rehabil 1999; 26: 624630. 